\title{
Article
}

\section{Effects of Higher Order Retarded Gravity}

\author{
Asher Yahalom ${ }^{1,2}$ (1) \\ 1 Ariel University, Faulty of Engineering, Department of Electrical \& Electronic Engineering, Ariel 40700, \\ Israel; asya@ariel.ac.il \\ 2 Ariel University, Center for Astrophysics, Geophysics, and Space Sciences (AGASS), Ariel 40700, Israel;
}

\begin{abstract}
In a recent paper we have a shown that the flattening of galactic rotation curves can be explained by retardation. However, this will rely on a temporal change of galactic mass. In our previous work we have kept only second order terms of the retardation time in our analysis, while higher terms in the Taylor expansion where not considered. Here we consider analysis to all orders and show that indeed a second order analysis will suffice, and higher order terms can be neglected.
\end{abstract}

Keywords: Galaxies; rotation curves; gravitational retardation;

\section{Introduction}

Dynamics of large scale structures is inconsistent with Newtonian mechanics. This was notified in the 1930's by Fritz Zwicky [1], who pointed out that if more (unseen) mass would be present one would be able to solve the apparent contradiction. The phenomena was also observed in galaxies by Volders [2] who have shown that star trajectories near the rim of galaxies do not move according to Newtonian predictions, and later corroborated by Rubin and Ford [3-5] for spiral galaxies.

In as series of papers we have shown that those discrepancies can be shown to result from retarded gravity as dictated by the theory of general relativity [6-10]. Indeed in the absence of temporal density changes, retardation does not effect the gravitational force. However, density is not constant for galaxies, in fact there are many processes that change the mass density in galaxies over time. Mass accretion from the inter galactic medium and internal processes such as super novae leading to super winds [10] modify the density. In addition to those local processes there is a cosmological decrease of density due to the cosmic expansion. However, the later process is many orders of magnitude smaller than the former.

In previous analysis [6-10] the corrected gravitational force was evaluated assuming a second order approximation in the retardation time $\frac{R}{c}$, neglecting higher order terms without justification. Here we take into account all orders and show that a second order approximation is indeed sufficient.

\section{Linear GR}

Only in cases of extreme compact objects (black holes and neutron stars) and the very early universe we consider the solution of the full non-linear Einstein Equations [6]. In most cases of astronomical interest (including the galactic case) a linear approximation to those equations around the flat Lorentz metric $\eta_{\mu v}$ is used, such that:

$$
g_{\mu v}=\eta_{\mu \nu}+h_{\mu \nu}, \quad \eta_{\mu \nu} \equiv \operatorname{diag}(1,-1,-1,-1), \quad\left|h_{\mu v}\right| \ll 1
$$

One then defines the quantity:

$$
\bar{h}_{\mu v} \equiv h_{\mu v}-\frac{1}{2} \eta_{\mu v} h, \quad h=\eta^{\mu v} h_{\mu v},
$$


$\bar{h}_{\mu v}=h_{\mu v}$ for non diagonal terms. For diagonal terms:

$$
\bar{h}=-h \Rightarrow h_{\mu v}=\bar{h}_{\mu v}-\frac{1}{2} \eta_{\mu v} \bar{h}
$$

It was shown ([11] page 75, exercise 37, see also [12-14]) that for a proper gauge the Einstein equations are:

$$
\square \bar{h}_{\mu v} \equiv \bar{h}_{\mu v, \alpha}{ }^{\alpha}=-\frac{16 \pi G}{c^{4}} T_{\mu v}, \quad \bar{h}_{\mu \alpha,}{ }^{\alpha}=0 .
$$

Equation (4) can be solved such that [15] :

$$
\begin{aligned}
& \bar{h}_{\mu v}(\vec{x}, t)=-\frac{4 G}{c^{4}} \int \frac{T_{\mu v}\left(\vec{x}^{\prime}, t-\frac{R}{c}\right)}{R} d^{3} x^{\prime}, \\
& t \equiv \frac{x^{0}}{c}, \quad \vec{x} \equiv x^{a} \quad a, b \in[1,2,3] \\
& \vec{R} \equiv \vec{x}-\vec{x}^{\prime}, \quad R=|\vec{R}| .
\end{aligned}
$$

In [16-19] we explain why the symmetry between space and time is broken, which justifies the use of different notations for space and time. $\frac{4 G}{c^{4}} \simeq 3.3 \times 10^{-44}$; is a tiny number, hence, in the above calculation one can take $T_{\mu \nu}$, to zeroth order in $h_{\alpha \beta}$. We now evaluate the affine connection in the linear approximation:

$$
\Gamma_{\mu v}^{\alpha}=\frac{1}{2} \eta^{\alpha \beta}\left(h_{\beta \mu, v}+h_{\beta v, \mu}-h_{\mu v, \beta}\right) .
$$

Notice that the affine connection has first order terms in $h_{\alpha \beta}$; hence, to the first order $\Gamma_{\mu \nu}^{\alpha} u^{\mu} u^{v}$ appearing in the geodesic equation, $u^{\mu} u^{v}$ must be taken to zeroth order. In which:

$$
u^{0}=\frac{1}{\sqrt{1-\frac{v^{2}}{c^{2}}}}, u^{a}=\vec{u}=\frac{\frac{\vec{v}}{c}}{\sqrt{1-\frac{v^{2}}{c^{2}}}}, \vec{v} \equiv \frac{d \vec{x}}{d t}, \quad v=|\vec{v}| .
$$

For velocities much smaller than the speed of light in vacuum:

$$
u^{0} \simeq 1, \quad \vec{u} \simeq \frac{\vec{v}}{c}, \quad u^{a} \ll u^{0} \quad \text { for } \quad v \ll c .
$$

Hence, the current paper does not discuss the post-Newtonian approximation, in which matter travels at speeds close to the speed of light, but we do consider the retardation effects which are due to the finite propagation speed of the gravitational field. We emphasize that assuming $\frac{v}{c}<<1$ is not the same as stating $\frac{R}{c}<<1$ (with $R$ being the typical size of a galaxy) since:

$$
\frac{R}{c}=\frac{v}{c} \frac{R}{v}
$$

now since in galaxies, $\frac{R}{v}$ is huge $\left(\frac{R}{v} \simeq 10^{15}\right.$ seconds); it follows that, $\frac{v}{c}$ can be dismissed but not $\frac{R}{c}$, for which $\frac{R}{c} \simeq 10^{12}$ seconds. Inserting Equations (6) and (8) in the geodesic equation, we arrive at the approximate equation:

$$
\frac{d v^{a}}{d t} \simeq-c^{2} \Gamma_{00}^{a}=-c^{2}\left(h_{0,0}^{a}-\frac{1}{2} h_{00}{ }^{a}\right)
$$

Taking a standard matter $T_{\mu \nu}$, assuming $\rho c^{2} \gg p$ and, taking into account Equation (8), we arrive at $T_{00}=\rho c^{2}$, while the remaining tensor components are much smaller. Therefore, $\bar{h}_{00}$ is larger than other components of $\bar{h}_{\mu v}$. Notice that it is not possible to deduce from the magnitudes of quantities that a similar difference exists between the derivatives of those quantities. Gauge conditions in Equation (4) lead to:

$$
\bar{h}_{\alpha 0,}{ }^{0}=-\bar{h}_{\alpha a,}{ }^{a} \quad \Rightarrow \bar{h}_{00}{ }^{0}=-\bar{h}_{0 a}{ }^{a}, \quad \bar{h}_{b 0,}{ }^{0}=-\bar{h}_{b a,}{ }^{a} .
$$


Thus, the zeroth derivative of $\bar{h}_{00}$ (which contains a $\frac{1}{c}$ ) is of similar order as the spatial derivative of $\bar{h}_{0 a}$. Also the zeroth derivative of $\bar{h}_{0 a}$ (see equation (10)) is of similar order as the spatial derivative of $\bar{h}_{a b}$. However, we can compare spatial derivatives of $\bar{h}_{00}$ and $\bar{h}_{a b}$ and conclude that the former is larger. Taking into account equation (3) and the above consideration, we write equation (10) as:

$$
\frac{d v^{a}}{d t} \simeq \frac{c^{2}}{4} \bar{h}_{00,}{ }^{a} \Rightarrow \frac{d \vec{v}}{d t}=-\vec{\nabla} \phi=\vec{F}, \quad \phi \equiv \frac{c^{2}}{4} \bar{h}_{00}
$$

Thus, the gravitational potential $\phi$ can be estimated using Equation (5):

$$
\begin{aligned}
\phi & =\frac{c^{2}}{4} \bar{h}_{00}=-\frac{G}{c^{2}} \int \frac{T_{00}\left(\vec{x}^{\prime}, t-\frac{R}{c}\right)}{R} d^{3} x^{\prime} \\
& =-G \int \frac{\rho\left(\vec{x}^{\prime}, t-\frac{R}{c}\right)}{R} d^{3} x^{\prime}
\end{aligned}
$$

and $\vec{F}$ is the force per unit mass. In the case that the mass density $\rho$ does not depend on time, we may use the Newtonian instantaneous action at a distance. Please notice that it is improbable that $\rho$ is static for a galaxy, as it accretes intergalactic medium gas.

Inserting equation (13) into equation (12) will lead to:

$$
\begin{aligned}
\vec{F} & =\vec{F}_{N r}+\vec{F}_{r} \\
\vec{F}_{N r} & =-G \int \frac{\rho\left(\vec{x}^{\prime}, t-\frac{R}{c}\right)}{R^{2}} \hat{R} d^{3} x^{\prime}, \quad \hat{R} \equiv \frac{\vec{R}}{R} \\
\vec{F}_{r} & \equiv-\frac{G}{c} \int \frac{\rho^{(1)}\left(\vec{x}^{\prime}, t-\frac{R}{c}\right)}{R} \hat{R} d^{3} x^{\prime}, \quad \rho^{(n)} \equiv \frac{\partial^{n} \rho}{\partial t^{n}} .
\end{aligned}
$$

Thus a retarded potential does not only imply a retarded Newtonian force $\vec{F}_{N r}$, but in addition a pure "retardation" force $\vec{F}_{r}$ which decreases slowly than the Newtonian force with distance, explaining the peculiar form of the galactic rotation curves. We emphasize that this result is independent of any perturbation expansion in the delay time $\frac{R}{c}$ as was done in [9]. However, the perturbation expansion does shed some light on the nature of those force terms as will be explained in the next section.

\section{Retardation Effects Beyond the Newtonian Approximation}

The duration $\frac{R}{c}$ may be tens of thousands of years, but may be short with respect to the duration in which the galactic density changes considerably. Thus, we write a Taylor series for the density:

$$
\rho\left(\vec{x}^{\prime}, t-\frac{R}{c}\right)=\sum_{n=0}^{\infty} \frac{1}{n !} \rho^{(n)}\left(\vec{x}^{\prime}, t\right)\left(-\frac{R}{c}\right)^{n} .
$$

By inserting Equations (15) into Equation (13), we will obtain:

$$
\begin{aligned}
\phi & =\phi_{2}+\phi_{(n>2)} \\
\phi_{2} & =-G \int \frac{\rho\left(\vec{x}^{\prime}, t\right)}{R} d^{3} x^{\prime}+\frac{G}{c} \int \rho^{(1)}\left(\vec{x}^{\prime}, t\right) d^{3} x^{\prime}-\frac{G}{2 c^{2}} \int R \rho^{(2)}\left(\vec{x}^{\prime}, t\right) d^{3} x^{\prime} \\
\phi_{(n>2)} & =-G \sum_{n=3}^{\infty} \frac{(-1)^{n}}{n ! c^{n}} \int R^{n-1} \rho^{(n)}\left(\vec{x}^{\prime}, t\right) d^{3} x^{\prime}
\end{aligned}
$$

The Newtonian potential is the first term, the second term has null contribution, and the third term is the lower order correction to the Newtonian theory:

$$
\phi_{r}=-\frac{G}{2 c^{2}} \int R \rho^{(2)}\left(\vec{x}^{\prime}, t\right) d^{3} x^{\prime}
$$


The expansion given in Equation (16), being a Taylor series expansion, is only valid for limited radii determined by the convergence of the infinite sum:

$$
R<c T_{\max } \equiv R_{\max }
$$

hence the current approximation can only be used in the near field regime, this is to be contrasted with the far field approximation used for gravitational radiation [28-30]. The restriction is even more severe when one uses a second order expansion as was done in [9].

If $n>2$ terms can be neglected the total force per unit mass can be approximated as:

$$
\begin{aligned}
\vec{F} & \simeq \vec{F}_{N}+\vec{F}_{a r} \\
\vec{F}_{N} & =-\vec{\nabla} \phi_{N}=-G \int \frac{\rho\left(\vec{x}^{\prime}, t\right)}{R^{2}} \hat{R} d^{3} x^{\prime}, \quad \hat{R} \equiv \frac{\vec{R}}{R} \\
\vec{F}_{a r} & \equiv-\vec{\nabla} \phi_{r}=\frac{G}{2 c^{2}} \int \rho^{(2)}\left(\vec{x}^{\prime}, t\right) \hat{R} d^{3} x^{\prime} .
\end{aligned}
$$

In the above $\vec{F}_{N}$ is a non retarded Newtonian force. To see how this comes about from the existence of a Newtonian retarded force and retardation force as defined in equation (14) we write those expressions up to order $O\left(1 / c^{2}\right)$ using equation (15), we thus have:

$$
\begin{aligned}
\vec{F}_{N r} & \simeq-G \int \frac{\rho\left(\vec{x}^{\prime}, t\right)}{R^{2}} \hat{R} d^{3} x^{\prime}+\frac{G}{c} \int \frac{\rho^{(1)}\left(\vec{x}^{\prime}, t\right)}{R} \hat{R} d^{3} x^{\prime}-\frac{G}{2 c^{2}} \int \rho^{(2)}\left(\vec{x}^{\prime}, t\right) \hat{R} d^{3} x^{\prime}, \\
\vec{F}_{r} & \simeq-\frac{G}{c} \int \frac{\rho^{(1)}\left(\vec{x}^{\prime}, t\right)}{R} \hat{R} d^{3} x^{\prime}+\frac{G}{c^{2}} \int \rho^{(2)}\left(\vec{x}^{\prime}, t\right) \hat{R} d^{3} x^{\prime} .
\end{aligned}
$$

Adding those two terms we see that the first order terms in $\frac{1}{c}$ cancel and we are left with the zeroth and second order terms which only partially cancel, as detailed in equation (19). The cancellation of first order terms is indeed remarkable as was pointed out by Feynman [20] with respect to the electromagnetic case.

$\vec{F}_{N}$ first introduced by Newton is attractive, however, the retardation force $\vec{F}_{r}$ can be either attractive or repulsive. Newtonian force decreases as $\frac{1}{R^{2}}$, however, the retardation force doe not depend on distance as long as the Taylor approximation given in Equation (15) holds. Below a certain distance, the Newtonian force dominates, but for larger distances the retardation force has the upper hand. Newtonian force can be neglected for distances significantly larger compared to the retardation distance:

$$
R \gg R_{r} \equiv c \Delta t
$$

$\Delta t$ is a duration associated with the second order derivative of the density $\rho$. For $R \ll R_{r}$, retardation can be neglected and only Newtonian forces need to be considered; this is the situation in the solar system. As the galaxy accretes intergalactic gas, the galactic mass becomes larger thus $\dot{M}>0$; however, the intergalactic gas is depleted, and thus the rate at which the mass is accreted decreases resulting in $\ddot{M}<0$, hence we have an attractive retardation force.

Some scholars have pointed out that since for the galaxy $\ddot{M}<0$ and the total mass is conserved it must be that $\ddot{M}>0$ for the matter outside the galaxy and thus retardation forces $\vec{F}_{a r}$ inside and outside the galaxy should cancel out. This derivation, however, is false because equation (19) is only valid when $\frac{R}{c}$ is small, it is certainly not small if the rest of the universe outside the galaxy is taken into account. We shall later show by a detailed model that a retardation force exist regardless if one assumes the expansion of equation (15) or not.

As a final comment to this section please notice that if we introduce dimensionless quantities using suitable dimensional constants, such that:

$$
\tilde{\rho} \equiv \frac{\rho}{\rho_{c}}, \quad \tilde{x} \equiv \frac{\vec{x}}{R_{s}}, \quad \bar{t}=\frac{t}{\Delta t}
$$


and define $\Lambda$ such that:

$$
\Lambda=\int \tilde{\rho} d^{3} \tilde{x}
$$

we obtain that:

$$
M=\int \rho d^{3} x=\rho_{c} R_{s}^{3} \int \tilde{\rho} d^{3} \tilde{x}=\Lambda \rho_{c} R_{s}^{3} .
$$

Thus $\vec{F}_{a r}$ given in equation (19) can be written in terms of the Schwarzschild radius $r_{s}=\frac{2 G M}{c^{2}}$ as follows:

$$
\vec{F}_{a r}=\frac{r_{s}}{\Delta t^{2}}\left[\frac{1}{4 \Lambda} \int \frac{\partial^{2} \tilde{\rho}}{\partial \bar{t}^{2}} \hat{R} d^{3} \tilde{x}^{\prime}\right] .
$$

the vector $\frac{1}{4 \Lambda} \int \frac{\partial^{2} \tilde{\rho}}{\partial \tilde{t}^{2}} \hat{R} d^{3} \tilde{x}^{\prime}$ is dimensionless.

\section{Higher order terms}

Comparing equation (31) to equation (82) of [9] it follows that:

$$
g^{(2)}(t)=g^{(2)}(0) e^{\frac{t}{\tau}}
$$

Hence:

$$
g^{(n)}(t)=g^{(2)}(0) \tau^{2-n} e^{\frac{t}{\tau}}=g^{(2)}(t) \tau^{2-n}, \quad n>2
$$

And also:

$$
\rho^{(n)}(\vec{x}, t)=\rho^{(2)}(\vec{x}, t) \tau^{2-n}, \quad M^{(n)}(t)=M^{(2)}(t) \tau^{2-n}, \quad n>2
$$

Thus according to equation (16) we have the following correction to the retardation potential:

$$
\phi_{(n>2)}=-G \sum_{n=3}^{\infty} \frac{(-1)^{n}}{n ! c^{n} \tau^{n-2}} \int R^{n-1} \rho^{(2)}\left(\vec{x}^{\prime}, t\right) d^{3} x^{\prime}
$$

The deviation from the second order approximation is more pronounced for large $r=|\vec{x}|$ for which $R=\left|\vec{x}-\vec{x}^{\prime}\right| \simeq r$ which is the case we consider here, thus:

$$
\phi_{(n>2)} \simeq-G \sum_{n=3}^{\infty} \frac{(-1)^{n}}{n ! c^{n} \tau^{n-2}} r^{n-1} \int \rho^{(2)}\left(\vec{x}^{\prime}, t\right) d^{3} x^{\prime}=-\frac{G \ddot{M}(t) \tau^{2}}{r} \sum_{n=3}^{\infty} \frac{1}{n !}\left(\frac{-r}{c \tau}\right)^{n} .
$$

Now using the well known identity:

$$
\sum_{n=3}^{\infty} \frac{\alpha^{n}}{n !}=e^{\alpha}-\left(1+\alpha+\frac{1}{2} \alpha^{2}\right)
$$

We may write equation (30) as a closed expression instead of an infinite sum:

$$
\phi_{(n>2)} \simeq-\frac{G \ddot{M}(t) \tau^{2}}{r}\left(e^{-\frac{r}{c \tau}}-1+\frac{r}{c \tau}-\frac{1}{2}\left(\frac{r}{c \tau}\right)^{2}\right) .
$$

For $r \ll c \tau$ it is quite clear that the term in the parenthesis of equation (32) vanishes, since:

$$
\lim _{\alpha \rightarrow 0} \sum_{n=3}^{\infty} \frac{\alpha^{n}}{n !}=\lim _{\alpha \rightarrow 0}\left(e^{\alpha}-\left(1+\alpha+\frac{1}{2} \alpha^{2}\right)\right)=0
$$

Hence $\phi_{(n>2)}$ can be neglected if indeed $r \ll c \tau$ for the relevant measurements of the M33 rotation curve, that is up to about $r<20 \mathrm{kpc}$. Now $\tau$ is dependent according to equation (81) of [9] on the density gradient of the inter galactic medium (IGM) and the typical velocity in this medium. Although those values are not known precisely we may assume that $v_{z} \sim 100 \mathrm{~km} / \mathrm{s}$ and the typical gradient is 
the same as the gradient of the optical disk luminosity that is $\frac{1}{k} \sim 0.1 \mathrm{kpc}$. Thus $\tau \sim 10^{6}$ years, and $\tau c \sim 300 \mathrm{kpc}$, making the second order approximation used so far reasonable.

\section{Retardation beyond the Taylor Expansion}

Although, we have shown in the previous section that second order expansion is sufficient in the framework of a particular galactic model, it is worthwhile to explore the domain of validity of the retardation phenomena in general. We have shown in section 3 that the retardation phenomena is not important at distances which are short with respect to the retardation length $r \ll R_{r}$, hence we need only to consider retardation at distances of about $r \geq \frac{R_{r}}{10}$. But is there an upper distance limit? Indeed the expansion treatment is valid only up to distances of $r<R_{\max }$, but this is a property of the Taylor expansion not of the retardation phenomena. We shall now show that indeed there is an upper distance $R_{r u l}$ beyond which the retardation phenomena is not important any more.

Let us look at the potential of equation (13) in the limit of large $r$ in which $r$ is much bigger than a typical scale of the system: $r \gg R_{s}$. In this limit $R \simeq r$ and thus the potential of equation (13) can be written as:

$$
\phi \simeq-G \int \frac{\rho\left(\vec{x}^{\prime}, t-\frac{r}{c}\right)}{r} d^{3} x^{\prime}=-\frac{G}{r} \int \rho\left(\vec{x}^{\prime}, t-\frac{r}{c}\right) d^{3} x^{\prime}=-\frac{G M}{r},
$$

in the above $M$ defined in equation (24) is the total mass of the system which is constant in time. The same analysis can be repeated for a Newtonian potential:

$$
\phi_{N} \simeq-G \int \frac{\rho\left(\vec{x}^{\prime}, t\right)}{r} d^{3} x^{\prime}=-\frac{G}{r} \int \rho\left(\vec{x}^{\prime}, t\right) d^{3} x^{\prime}=-\frac{G M}{r},
$$

it follows that for large distances such that $r \gg R_{S}$ it makes not difference if we use a Newtonian or retarded potential, hence "dark matter" effects disappear. The above is only true for an isolated system that does exchange mass with its environment. For a galaxy this will include its "sphere of influence" which is not limited to the observable galaxy but includes also a surrounding from which the observable galaxy accretes mass from. Mathematically we may define:

$$
\Delta \phi \equiv \phi-\phi_{N}
$$

Thus:

$$
\lim _{r \rightarrow \infty} \Delta \phi=\lim _{r \rightarrow>\infty}\left(\phi-\phi_{N}\right)=0,
$$

indicating that there is an upper scale $R_{r u l} \simeq 10 R_{S}$ above which $r>R_{r u l}$ retardation effects are unimportant. We can summarize the range of validity of the retardation phenomena by the inequality:

$$
\frac{R_{r}}{10}<r<10 R_{S}
$$

leading us to expect no retardation phenomena for systems in which:

$$
R_{r}>100 R_{s} .
$$

To study the phenomena of retardation within a specific model we write the potential of equation (13) using dimensionless coordinates:

$$
\phi=-G \frac{\rho_{c} R_{s}^{3}}{r} r \int \frac{\tilde{\rho}\left(\vec{x}^{\prime}, t-\frac{R}{c}\right)}{R} d^{3} \tilde{x}^{\prime}=-\frac{G M}{r} \frac{1}{\Lambda} \int \frac{r}{R} \tilde{\rho}\left(\vec{x}^{\prime}, t-\frac{R}{c}\right) d^{3} \tilde{x}^{\prime}
$$

in which we used equation (24). This leads to the definition:

$$
\psi_{r} \equiv \frac{1}{\Lambda} \int \frac{r}{R} \tilde{\rho}\left(\vec{x}^{\prime}, t-\frac{R}{c}\right) d^{3} \tilde{x}^{\prime} \quad \Rightarrow \quad \phi=-\frac{G M}{r} \psi_{r}
$$


Similarly we define a Newtonian $\psi_{N}$ :

$$
\psi_{N} \equiv \frac{1}{\Lambda} \int \frac{r}{R} \tilde{\rho}\left(\vec{x}^{\prime}, t\right) d^{3} \tilde{x}^{\prime} \quad \Rightarrow \quad \phi_{N}=-\frac{G M}{r} \psi_{N} .
$$

Now according to equation (34) and equation (35):

$$
\lim _{r \rightarrow \infty} \psi_{N}=\lim _{r \rightarrow \infty} \psi_{r}=1
$$

I thus follows that:

$$
\Delta \psi \equiv \psi_{r}-\psi_{N} \quad \Rightarrow \quad \lim _{r \rightarrow \infty} \Delta \psi=0
$$

And also according to equation (36):

$$
\Delta \phi=-\frac{G M}{r} \Delta \psi
$$

\section{A Specific Model}

Let us now study the effects of retardation in the framework of a specific models:

$$
\rho=\rho_{c} \tilde{\rho}, \quad \tilde{\rho}=\Sigma\left(\vec{x}_{\perp}\right) h(z, t)
$$

in which $\Sigma\left(\vec{x}_{\perp}\right)$ is dependent on the coordinates in the transversal direction $\vec{x}_{\perp}$ and $h$ is dependent on the time $t$ and on the coordinates along the axial direction $z$. We will further assume that:

$$
\Sigma\left(\vec{x}_{\perp}\right)=\delta(\tilde{x}) \delta(\tilde{y})
$$

in which $\delta$ is a Dirac delta function. It is clear that any profile can be constructed from a weighted sum of $\Sigma\left(\tilde{x}-\tilde{x}_{i}, \tilde{y}-\tilde{y}_{i}\right)$ located at different $\left(\tilde{x}_{i}, \tilde{y}_{i}\right)$. For the axial direction we assume a Gaussian profile:

$$
h(z, t)=\frac{R_{s}}{\sqrt{2 \pi} \sigma(t)} e^{-\frac{z^{2}}{2 \sigma(t)^{2}}} .
$$

The time dependence is given through the width of the Gaussian profile which assumes the following form:

$$
\sigma(t)=\left\{\begin{array}{cc}
\sigma_{i} & \bar{t} \leq 0 \\
\sigma_{i}+\left(\sigma_{f}-\sigma_{i}\right) \bar{t}(2-\bar{t}) & 0<\bar{t}<1 \\
\sigma_{f} & \bar{t} \geq 1
\end{array} \quad \bar{t} \equiv \frac{t}{t_{f}}\right.
$$

$t_{f}$ is a typical time scale, $\sigma_{i}$ is the initial time width before a change takes place and $\sigma_{f}$ is the resulting time width after the change took place. We shall define

$$
\tilde{\sigma}(t)=\frac{\sigma(t)}{R_{s}}, \quad \tilde{\sigma}_{i}=\frac{\sigma_{i}}{R_{s}}, \quad \tilde{\sigma}_{f}=\frac{\sigma_{f}}{R_{s}} .
$$

Hence:

$$
h(z, t)=\frac{1}{\sqrt{2 \pi} \tilde{\sigma}(t)} e^{-\frac{\tilde{z}^{2}}{2 \tilde{\sigma}(t)^{2}}} .
$$

Choosing conveniently $\sigma_{f}=R_{S}$ and $\sigma_{i}=1.2 R_{s}$ we obtain:

$$
\tilde{\sigma}(t)=\left\{\begin{array}{cc}
1.2 & \bar{t} \leq 0 \\
1.2-0.2 \bar{t}(2-\bar{t}) & 0<\bar{t}<1 \\
1.0 & \bar{t} \geq 1
\end{array}\right.
$$

The profile width evolution is depicted in figure 1. 
8 of 15

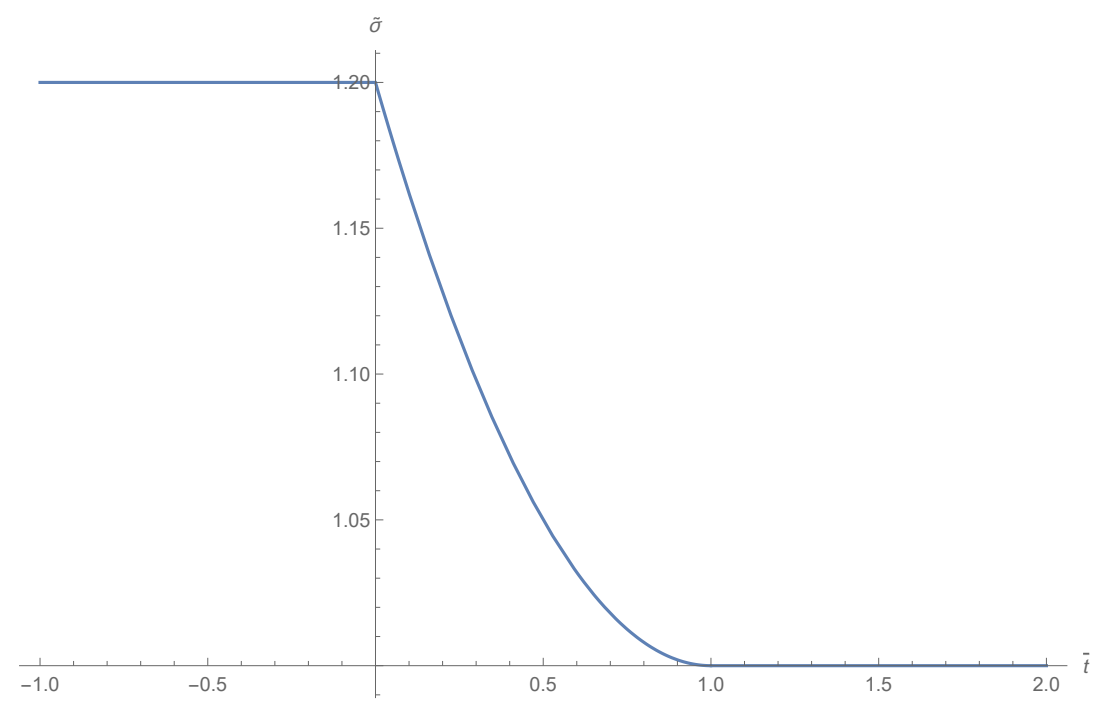

Figure 1. The profile width evolution.

The axial density profile $h(z, t)$ is depicted for $\bar{t}=0$ and $\bar{t}=1$ in figure 2 , and for any time in between in figure 3

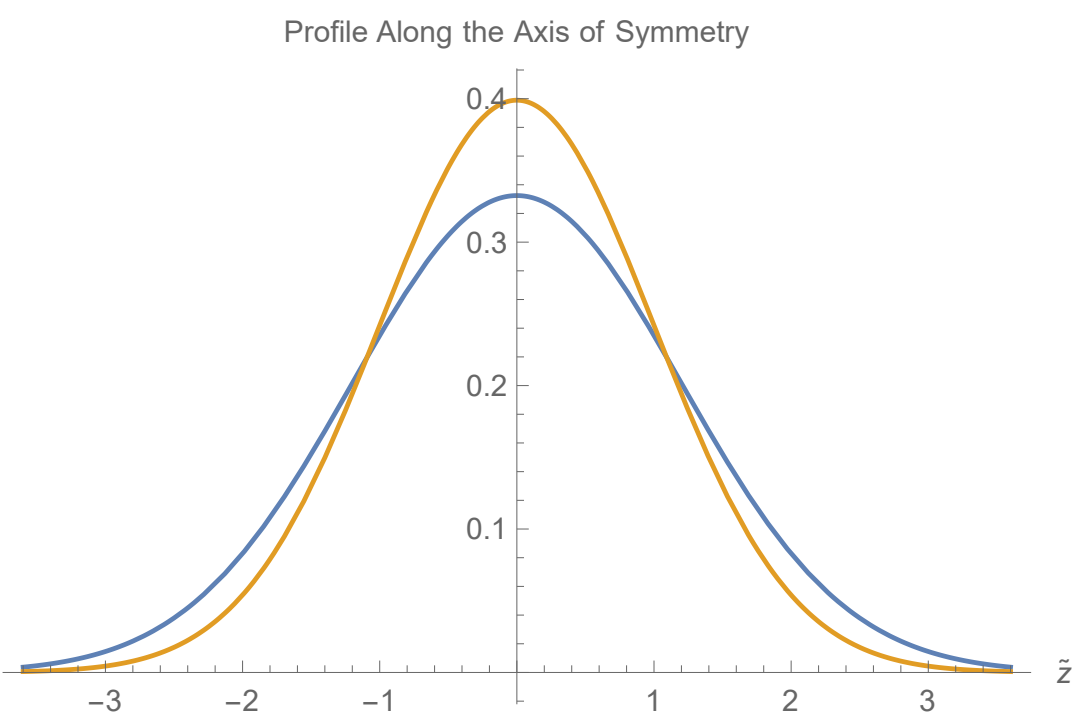

Figure 2. Axial density profile for $\bar{t}=0$ and $\bar{t}=1$. 


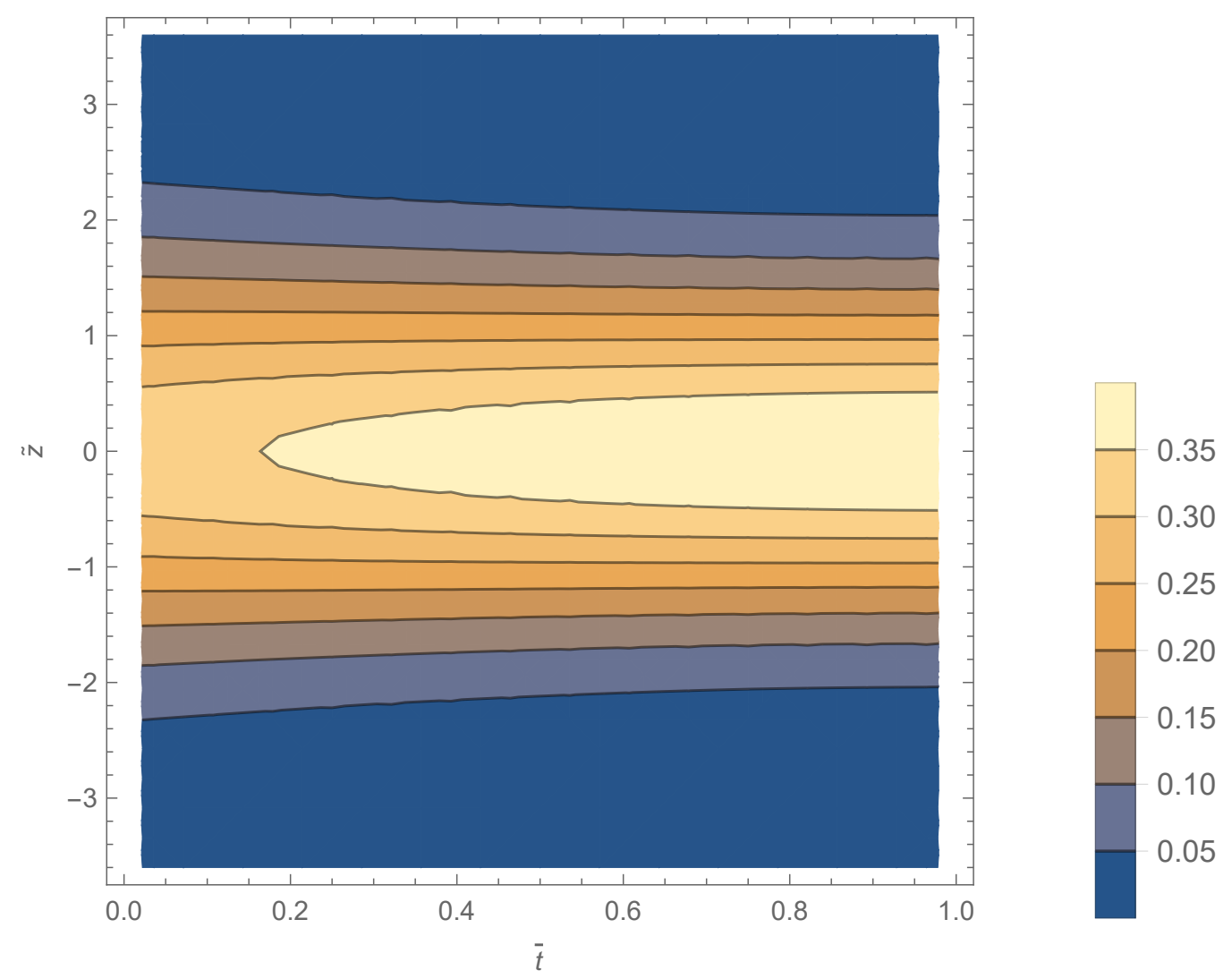

Figure 3. Axial density profile between $\bar{t}=0$ and $\bar{t}=1$.

We note that for the current profile the mass $M$ is constant although the density profile is time dependent, we also note that for the current density profile we have $\Lambda=1$ according to equation (23).

We are now in a position to calculate $\psi_{N}$ and $\psi_{r}$ using equation (46), equation (47) and equation (51). We shall choose a point of distance $r$ from the origin along the $x$ axis located at the $z=0$ plane to evaluate $\psi$, since the density profile is cylindrically symmetric every point in the $z=0$ plane of distance $r$ to the origin is equivalent to any other. Taking into account equation (42) we have:

$$
\psi_{N}(\tilde{r}, \bar{t})=\int \frac{\tilde{r}}{\tilde{R}} h\left(\tilde{z}^{\prime}, \bar{t}\right) d \tilde{z}^{\prime}, \quad \tilde{R}=\sqrt{\tilde{z}^{\prime 2}+\tilde{r}^{2}}
$$

The function $\psi_{N}(\tilde{r}, 0.5)$ is depicted in figure 4 in which it is clear that $\psi_{N}$ asymptotically approaches 1 . 


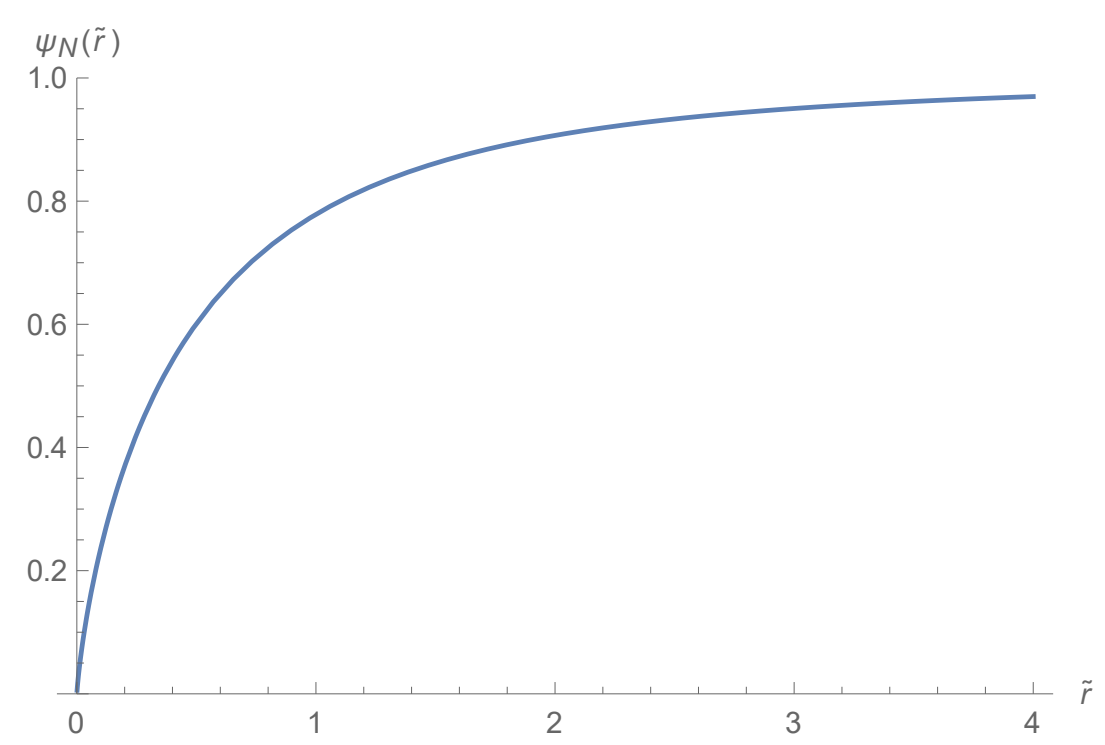

Figure 4. The function $\psi_{N}(\tilde{r}, 0.5)$, it is clear that $\psi_{N}$ approaches 1 asymptotically.

Taking into account equation (41) we have:

$$
\psi_{r}(\tilde{r}, \bar{t})=\int \frac{\tilde{r}}{\tilde{R}} h\left(\tilde{z}^{\prime}, \bar{t}-\frac{R}{c t_{f}}\right) d \tilde{z}^{\prime} .
$$

Hence we have another length scale $R_{s 2}=c t_{f}$ which is conveniently chosen to be $R_{s 2}=2 R_{s}$. The function $\psi_{r}(\tilde{r}, 0.5)$ is depicted in figure 5 in which it is clear that $\psi_{r}$ also asymptotically approaches 1 .

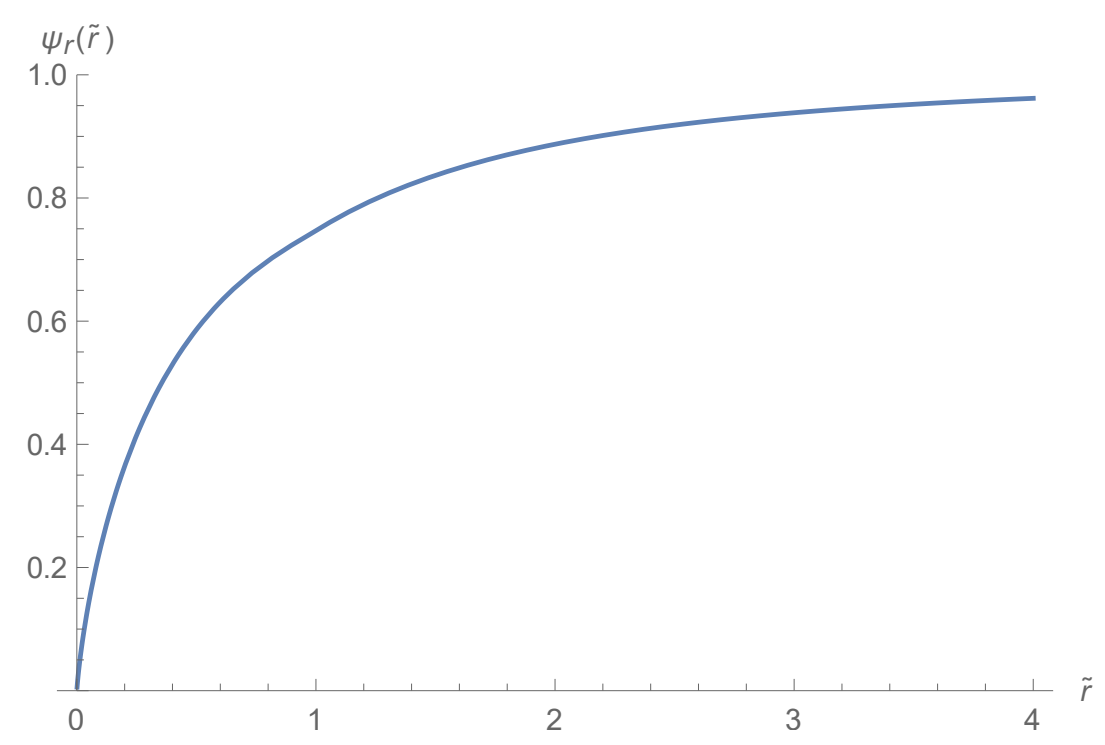

Figure 5. The function $\psi_{r}(\tilde{r}, 0.5)$, it is clear that $\psi_{r}$ approaches 1 asymptotically.

The difference between the two functions $\Delta \psi$ is defined in equation (44) and depicted in figure 6. It is clear that the difference exist and does not depend on a Taylor expansion, it also clear that this difference may be approximated by a simple function to about $\tilde{r}=1$, or starting at $\tilde{r}=1$ and using a simple function to describe the function outwards which would work for at least $\tilde{r}=4$. To see that the function $\Delta \psi$ indeed approached 0 asymptotically as predicted by equation (44) the $\tilde{r}$ axis is extended to $\tilde{r}=20$ as depicted in figure 7 . 


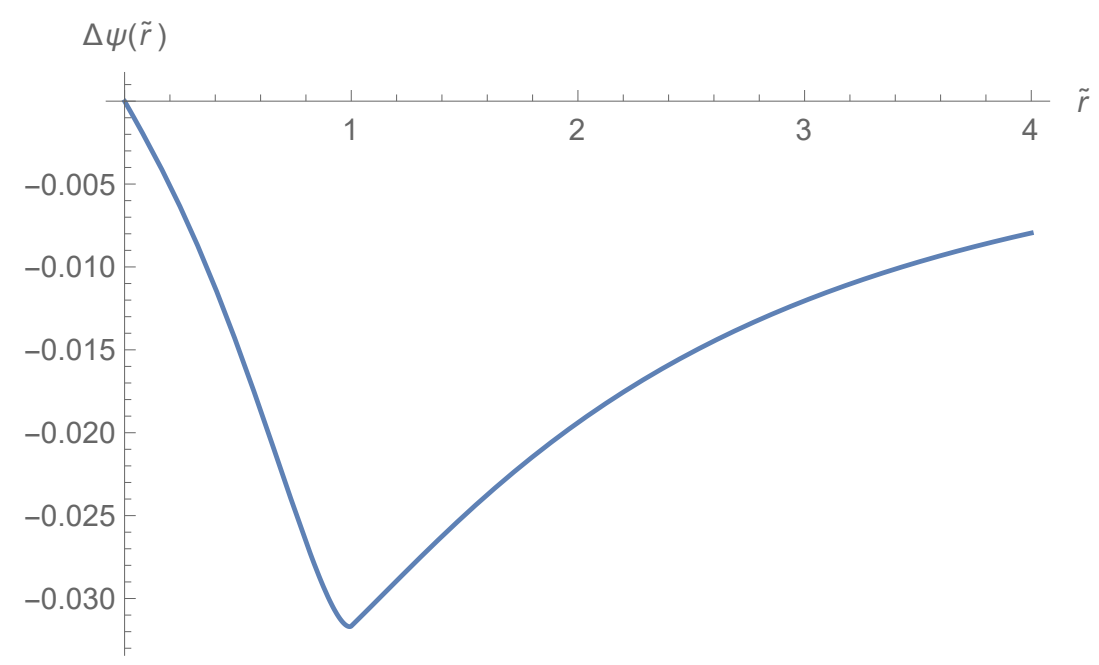

Figure 6. The function $\Delta \psi(\tilde{r}, 0.5)$ depicted up to $\tilde{r}=4$.

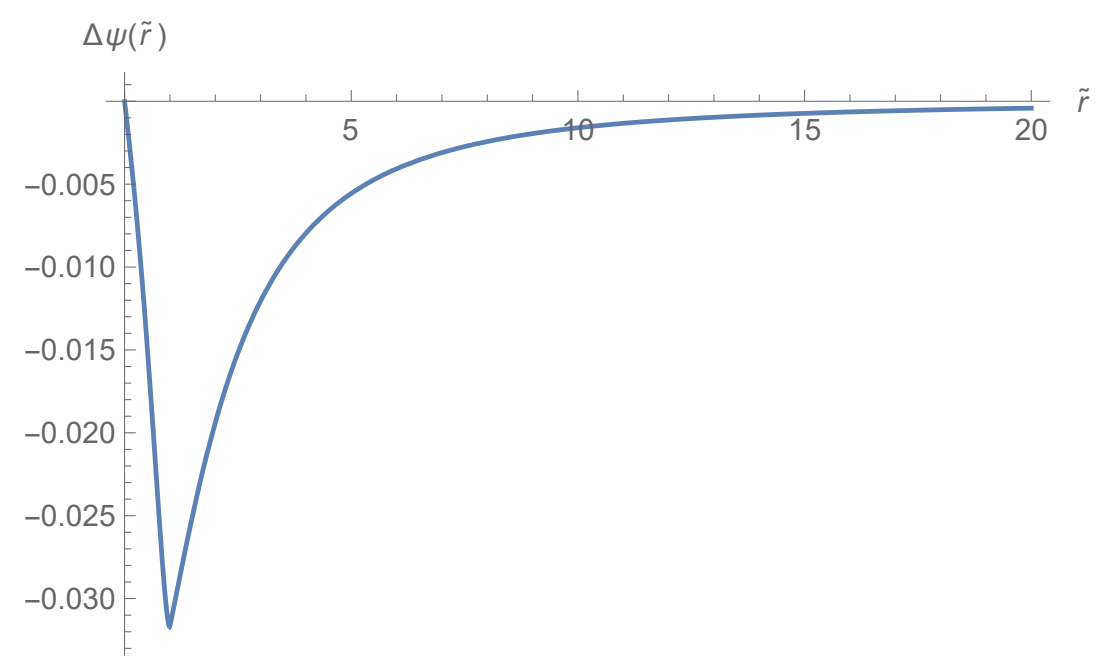

Figure 7. The function $\Delta \psi(\tilde{r}, 0.5)$ depicted up to $\tilde{r}=20$.

To conclude this section we shall discuss the applicability of a Taylor expansion of the type used in equation (15). In the current context, we study the function $h(\tilde{z}, \bar{t})$, that is we would like to know how good is the approximation:

$$
\Delta h(\tilde{z}, \bar{t}, \Delta \bar{t}) \equiv h(\tilde{z}, \bar{t}+\Delta \bar{t})-h(\tilde{z}, \bar{t}) \simeq \Delta h_{a}(\tilde{z}, \bar{t}, \Delta \bar{t}), \quad \Delta h_{a}(\tilde{z}, \bar{t}, \Delta \bar{t}) \equiv \Delta \bar{t} h^{(1)}(\tilde{z}, \bar{t})+\frac{1}{2} \Delta \bar{t}^{2} h^{(2)}(\tilde{z}, \bar{t})
$$

The numerical evaluation of the functions $\Delta h(\tilde{z}, \bar{t}, \Delta \bar{t})$ and $\Delta h_{a}(\tilde{z}, \bar{t}, \Delta \bar{t})$ are depicted in figure 8 for the plane $z=0$ and for the time $\bar{t}=0.5$. It is easy to see that the approximation is indeed a good one for $|\Delta \bar{t}|<0.5$, otherwise the approximation is not valid especially for a negative $\Delta \bar{t}$ which is our main concern in retardation theory. 


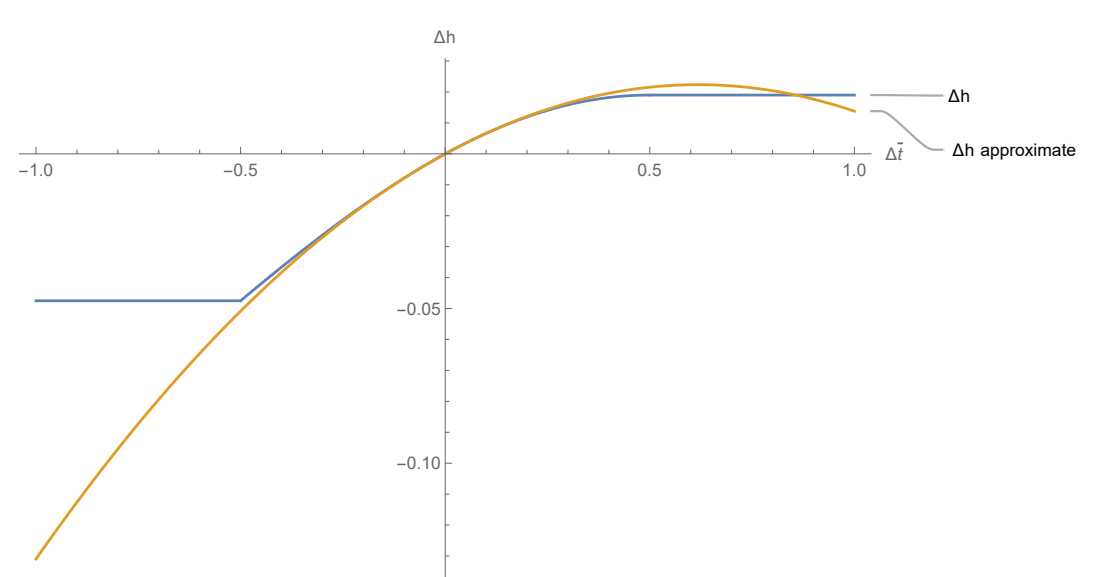

Figure 8. The functions $\Delta h(0,0.5, \Delta \bar{t})$ and $\Delta h_{a}(0,0.5, \Delta \bar{t})$.

The next step is to set the retardation delay $\Delta \bar{t}=-\frac{R}{c t_{f}}=-\frac{R}{R_{s 2}}=-\frac{\tilde{R}}{\tilde{R}_{s 2}}$ and calculate the approximate form of $\Delta \psi$ :

$$
\Delta \psi(r, \bar{t})=\psi_{r}(r, \bar{t})-\psi_{N}(r, \bar{t})=\int \frac{\tilde{r}}{\tilde{R}} \Delta h\left(\tilde{z}^{\prime}, \bar{t},-\frac{\tilde{R}}{\tilde{R}_{s 2}}\right) d \tilde{z}^{\prime} \simeq \Delta \psi_{a}(r, \bar{t}) .
$$

We may try the following approximation:

$$
\Delta \psi_{a w}(r, \bar{t}) \equiv \int_{-\infty}^{\infty} \frac{\tilde{r}}{\tilde{R}} \Delta h_{a}\left(\tilde{z}^{\prime}, \bar{t},-\frac{\tilde{R}}{\tilde{R}_{s 2}}\right) d \tilde{z}^{\prime}=-\frac{\tilde{r}}{\tilde{R}_{s 2}} \int_{-\infty}^{\infty} h^{(1)}\left(\tilde{z}^{\prime}, \bar{t}\right) d \tilde{z}^{\prime}+\frac{\tilde{r}}{2 \tilde{R}_{s 2}} \int_{-\infty}^{\infty} \frac{\tilde{R}}{\tilde{R}_{s 2}} h^{(2)}\left(\tilde{z}^{\prime}, \bar{t}\right) d \tilde{z}^{\prime}
$$

However, notice that for many points in the integration domain $\left|\frac{\tilde{R}}{\tilde{R}_{s 2}}\right|$ is not smaller than $\frac{1}{2}$ hence $\Delta h_{a}$ is not a valid approximation. Moreover, notice that:

$$
\int_{-\infty}^{\infty} h^{(1)}\left(\tilde{z}^{\prime}, \bar{t}\right) d \tilde{z}^{\prime}=\frac{\partial}{\partial \bar{t}} \int_{-\infty}^{\infty} h\left(\tilde{z}^{\prime}, \bar{t}\right) d \tilde{z}^{\prime}=\frac{\partial}{\partial \bar{t}} 1=0
$$

Hence:

$$
\Delta \psi_{a w}(\tilde{r}, \bar{t})=\frac{\tilde{r}}{2 \tilde{R}_{s 2}} \int_{-\infty}^{\infty} \frac{\tilde{R}}{\tilde{R}_{s 2}} h^{(2)}\left(\tilde{z}^{\prime}, \bar{t}\right) d \tilde{z}^{\prime}
$$

However, $\Delta \psi_{a w}(r, \bar{t})$ does not have the asymptotic property $\lim _{r->\infty} \Delta \psi_{a w}(r, \bar{t})=0$ described in equation (44) as can be seen by a numerical evaluation presented in figure 9 . We conclude that $\Delta \psi_{a w}$ is not an adequate approximation of $\Delta \psi$. 


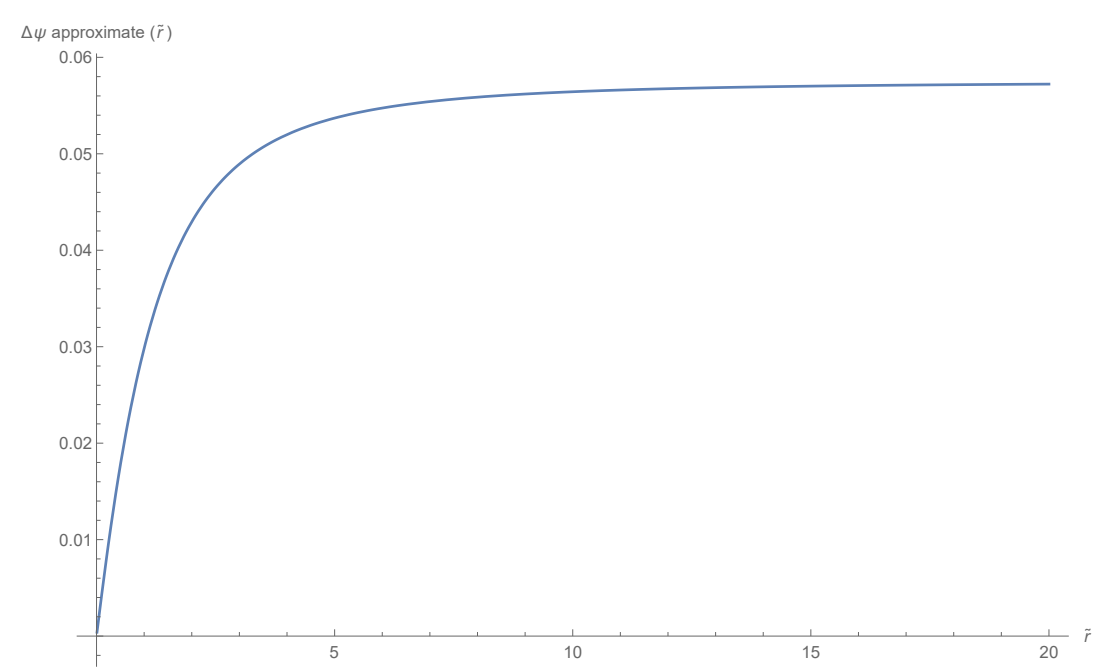

Figure 9. The asymptotic behaviour of $\Delta \psi_{a w}(\tilde{r}, \bar{t})$ is clearly not appropriate.

Notice, however, that this problem can be somewhat elevated if one integrates close enough to the galactic plane $z=0$ such that $\left|\frac{\tilde{R}}{\tilde{R}_{s 2}}\right|<0.5$ and consider small $\tilde{r}$. Indeed, most of the mass in centered close to the galactic plane. After some numerical experimentation we obtained a reasonable approximation integrating in the range: $\left[-0.25 \tilde{\sigma}_{i}, 0.25 \tilde{\sigma}_{i}\right]$ :

$\Delta \psi_{a c}(r, \bar{t}) \equiv \int_{-0.25 \tilde{\sigma}_{i}}^{0.25 \tilde{\sigma}_{i}} \frac{\tilde{r}}{\tilde{R}} \Delta h_{a}\left(\tilde{z}^{\prime}, \bar{t},-\frac{\tilde{R}}{\tilde{R}_{s 2}}\right) d \tilde{z}^{\prime}=-\frac{\tilde{r}}{\tilde{R}_{s 2}} \int_{-0.25 \tilde{\sigma}_{i}}^{0.25 \tilde{\sigma}_{i}} h^{(1)}\left(\tilde{z}^{\prime}, \bar{t}\right) d \tilde{z}^{\prime}+\frac{\tilde{r}}{2 \tilde{R}_{s 2}} \int_{-0.25 \tilde{\sigma}_{i}}^{0.25 \tilde{\sigma}_{i}} \frac{\tilde{R}}{\tilde{R}_{s 2}} h^{(2)}\left(\tilde{z}^{\prime}, \bar{t}\right) d \tilde{z}^{\prime}$.

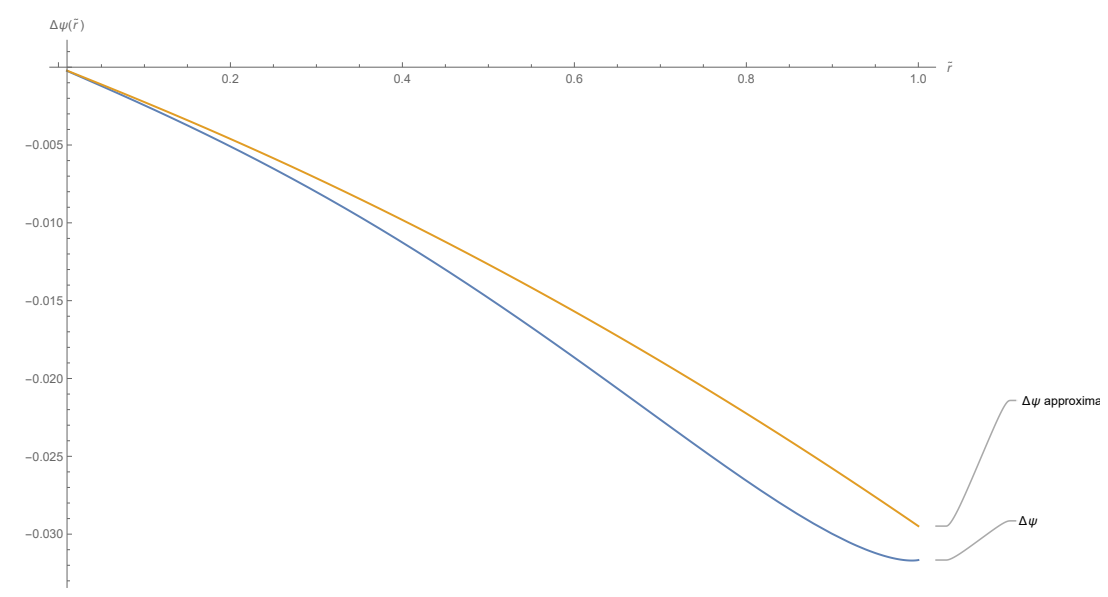

Figure 10. $\Delta \psi(\tilde{r}, 0.5)$ and $\Delta \psi_{a c}(\tilde{r}, 0.5)$ for $\tilde{r}<1$

As a final comment we stress again that $R_{S}$ is the scale of the "galactic sphere of influence" that is the typical dimension of the region with which the galaxy exchanges mass and not necessarily the galactic radius. Thus the domain $\tilde{r}<1$ may stretch far beyond the galaxy itself.

\section{Conclusions}

The phenomena of retardation is ubiquitous in physics, and follows directly from the Lorentz symmetry group. Hence, any system that is invariant under the Lorentz transformation will exhibit retardation phenomena. Those include physical systems related to classical electromagnetism [21-24] General Relativity [6-9], but also to other Lorentz invariant theories such as conformal gravity [25-27].

Dark matter being a major candidate to explain galactic rotation curves has only a slim chance to being found, given that accelerator experiments, as using the Large Hadron Collider was unable to find any super symmetric particles, not only of the community's favorite form of dark matter, but also 
the form of it that is mandated in string theory, a theory that also suggests a quantized version of Einstein gravity.

We have shown that at least on the galactic scale dark matter is not needed [6-10], as the dynamics can be explained by a retarded gravitational potential when a near field approximation is used. We remark that the analysis of far field leading to gravitational waves [28] was corroborated in recent years by observations $[29,30]$.

A justification for the second order Taylor series approximation which we used in previous works is given here for the first time, showing that indeed higher order terms can be safely neglected.

We also show that one may discuss retardation phenomena without relying on a Taylor expansion.

Finally we mention other approaches to the galactic rotation curves problem, which suggest to overcome the problem by changing the laws of gravity. Such approaches are Milgrom's MOND [31] and Mannheim's conformal gravity [25-27]. Unfortunately those approaches seem to contradict both general relativity (which is supported by a large body of observational evidence) and recent observations. Indeed van Dokkum et al. [32] have shown that there are galaxies with Newtonian rotation curves, excluding the possibility of a universal modification to the laws of gravity which prevail in every galaxy. Thus either van Dokkum's galaxy is devoid of "dark matter" or it has a small retardation depletion effect as the gas around it has not yet depleted (or fully depleted).

Given the negative results from accelerator experiments regarding dark matter, retardation theory seem to be the only valid option.

Author Contributions: This paper has a single author, which has done all the reported work.

Funding: This research received no funding.

Acknowledgments: The author wishes to thank his former student, Dr. Michal Wagman, for being critical regarding retardation theory. Much of this paper is a result of her raising various questions on the validity of the second order approximation which was used in [9]. The same doubts were raised by my colleague and friend Prof. Yosef Pinhasi, I would like to thank him as well for a critical discussion.

Conflicts of Interest: The author declares no conflict of interest.

\section{References}

1. Zwicky, F. On a New Cluster of Nebulae in Pisces. Proc. Natl. Acad. Sci. USA 1937, 23, 251-256.

2. Volders, L.M.J.S. Neutral Hydrogen in M33 and M101. Bull. Astr. Inst. Netherl. 1959, 14, 323.

3. Rubin, V.C.; Ford, W.K., Jr. Rotation of the Andromeda Nebula from a Spectroscopic Survey of Emission Regions. Astrophys. J. 1970, 159, 379.

4. Rubin, V.C.; Ford, W.K., Jr.; Thonnard, N. Rotational Properties of $21 \mathrm{Sc}$ Galaxies with a Large Range of Luminosities and Radii from NGC 4605 ( $\mathrm{R}=4 \mathrm{kpc}$ ) to UGC 2885 ( $\mathrm{R}=122 \mathrm{kpc})$. Astrophys. J. 1980, 238, 471.

5. Binney, J.; \& Tremaine, S. Galactic Dynamics; Princeton University Press: Princeton, NJ, USA, 1987.

6. Yahalom, A. The effect of Retardation on Galactic Rotation Curves. J. Phys.: Conf. Ser. 1239 (2019) 012006.

7. Yahalom, A. Retardation Effects in Electromagnetism and Gravitation. In Proceedings of the Material Technologies and Modeling the Tenth International Conference, Ariel University, Ariel, Israel, 20-24 August 2018. (arXiv:1507.02897v2)

8. Yahalom, A. Dark Matter: Reality or a Relativistic Illusion? In Proceedings of Eighteenth Israeli-Russian Bi-National Workshop 2019, The Optimization of Composition, Structure and Properties of Metals, Oxides, Composites, Nano and Amorphous Materials, Ein Bokek, Israel, 17-22 February 2019.

9. Asher Yahalom "Lorentz Symmetry Group, Retardation, Intergalactic Mass Depletion and Mechanisms Leading to Galactic Rotation Curves" Symmetry 2020, 12(10), 1693.

10. Wagman, M. Retardation Theory in Galaxies. Ph.D. Thesis, submitted to the Senate of Ariel University, Ariel, Israel, 23 September 2019.

11. Narlikar, J.V. Introduction to Cosmology, 2nd ed.; Cambridge University Press: Cambridge, UK, 1993.

12. Eddington, A.S. The Mathematical Theory of Relativity; Cambridge University Press: Cambridge, UK, 1923.

13. Weinberg, S. Gravitation and Cosmology: Principles and Applications of the General Theory of Relativity; John Wiley \& Sons, Inc.: Hoboken, NJ, USA, 1972.

14. Misner, C.W.; Thorne, K.S.; Wheeler, J.A. Gravitation; W.H. Freeman \& Company: New York, NY, USA, 1973. 
15. Jackson, J.D. Classical Electrodynamics, 3rd ed.; Wiley: New York, NY, USA, 1999.

16. Yahalom, A. The Geometrical Meaning of Time. Found. Phys. 2008, 38, 489-497.

17. Yahalom, A. The Gravitational Origin of the Distinction between Space and Time. Int. J. Mod. Phys. D 2009, 18, 2155-2158.

18. Asher Yahalom "Gravity and the Complexity of Coordinates in Fisher Information" International Journal of Modern Physics D, Vol. 19, No. 14 (2010) 2233-2237, (c)World Scientific Publishing Company DOI: 10.1142/S0218271810018347.

19. Asher Yahalom "Gravity, Stability and Cosmological Models". International Journal of Modern Physics D. Published: 10 October 2017 issue (No. 12). https:/ / doi.org/10.1142/S021827181717026X

20. R. P. Feynman, R. B. Leighton \& M. L. Sands, Feynman Lectures on Physics, Basic Books; revised 50th anniversary edition (2011).

21. Tuval, M.; Yahalom, A. Newton's Third Law in the Framework of Special Relativity. Eur. Phys. J. Plus 2014, 129, 240, doi:10.1140/epjp/i2014-14240-x.

22. Tuval, M.; Yahalom, A. Momentum Conservation in a Relativistic Engine. Eur. Phys. J. Plus 2016, 131, 374, doi:10.1140/epjp/i2016-16374-1.

23. Yahalom, A. Retardation in Special Relativity and the Design of a Relativistic Motor. Acta Phys. Pol. A 2017, 131, 1285-1288.

24. Shailendra Rajput, Asher Yahalom \& Hong Qin "Lorentz Symmetry Group, Retardation and Energy Transformations in a Relativistic Engine" Symmetry 2021, 13, 420. https:/ /doi.org/10.3390/sym13030420.

25. Mannheim, P.D. \& Kazanas, D. Exact vacuum solution to conformal Weyl gravity and galactic rotation curves Astrophys. J. 1989, 342, 635.

26. Mannheim, P.D. Linear Potentials and Galactic Rotation Curves Astrophys. J. 1993, 149, 150.

27. Mannheim, P.D. Are Galactic Rotation Curves Really Flat? Astrophys. J. 1997, 479, 659.

28. Einstein, A. Näherungsweise Integration der Feldgleichungen der Gravitation. Sitzungsberichte der Königlich Preussischen Akademie der Wissenschaften Berlin; Part 1; 1916; pp. 688-696. The Prusssian Academy of Sciences, Berlin, Germany.

29. Nobel Prize, A. Press Release The Royal Swedish Academy of Sciences; 1993.The Royal Swedish Academy of Sciences, Stockholm, Sweden.

30. Castelvecchi, D.; Witze, W. Einstein's gravitational waves found at last. Nature News 2016, doi:10.1038/nature.2016.19361.

31. Milgrom, M. A modification of the Newtonian dynamics as a possible alternative to the hidden mass hypothesis. Astrophys. J. 1983, 270, 365-370, doi:10.1086/161130.

32. van Dokkum, P.; Danieli, S.; Cohen, Y.; Merritt, A.; Romanowsky, A.J.; Abraham, R.; Brodie, J.; Conroy, C.; Lokhorst, D.; Mowla, L.; et al. A galaxy lacking dark matter. Nature 2018, 555, 629-632, doi:10.1038/nature25767. 